\title{
SPI for the JINR accelerator complex
}

\author{
V. V. Fimushkin*, A. D. Kovalenko, L. V. Kutuzova, Yu. V. Prokofichev, V. B. Shutov \\ Institute for Nuclear Research, Joliot-Curie 6, 141980 Dubna, Moscow region, Russia. \\ E-mail: fimushkinejinr.ru
}

\section{A. S. Belov, V. N. Zubets and A. V. Turbabin}

Institute for Nuclear Research of RAS, prospect 60letiya Oktyabrya, 7A, 117312, Moscow, Russia

\begin{abstract}
The article describes the status of the JINR polarized ion source development. The source is under testing at the test-bench of LHEP, JINR. The charge-exchange plasma ionizer has been tested at first without a storage cell in the ionization region. The deuterium plasma source produced plasma jet in the ionization region. The unpolarized deuterium ion beam with peak current of $160 \mathrm{~mA}$, $22 \mathrm{keV}$ energy, pulse duration of $100 \mu \mathrm{s}$ and repetition rate of $1 \mathrm{~Hz}$ has been extracted from the ionization region. The polarized proton beam with peak current of $1.4 \mathrm{~mA}$ has been obtained with a free polarized atomic hydrogen beam injected into the ionizer. The future source development includes tests of the ionizer with the storage cell and tuning of the high frequency transition units by means of a Breit-Rabi polarimeter.
\end{abstract}

XVIth International Workshop in Polarized Sources, Targets, and Polarimetry, PSTP2015,

14-18 September 2015,

Bochum, Germany

* Speaker. 


\section{Introduction}

The high intensity pulsed source of polarized ions (SPI) has been developed at JINR in collaboration with INR RAS to ensure injection of polarized deuterons and protons into the Nuclotron and a future collider of heavy and light ions (NICA project) [1]. The SPI works on the atomic beam method using the charge-exchange plasma ionizer and a storage cell in it $[2,3,4]$. Some parts of the polarized ion source CIPIOS from IUCF [5] are included in the project.

\section{General description of the polarized ion source}

A schematic diagram of the SPI is shown in Figure 1. The source has been described in detail in [6]. Atomic hydrogen (or deuterium) for polarized deuterons production is generated in the source using an RF discharge dissociator. The dissociator operates in a pulsed mode with a repetition rate up to $1 \mathrm{~Hz}$ and RF discharge duration of up to $3 \mathrm{~ms}$. Two electromagnetic valves are used for pulsed supply of gas into the dissociator discharge tube: one of the valves is applied to inject the gaseous molecular hydrogen (deuterium) and the other one - for injecting molecular oxygen. The atomic deuterium is cooled while passing through the pyrex tube having a $5 \mathrm{~mm}$ internal diameter with a sonic nozzle of $2.3 \mathrm{~mm}$ in diameter at the exit. The channel walls are cooled down to the temperature of $80 \mathrm{~K}$ by means of a cryocooler (model 350 Cryodyne refrigerator system). A skimmer is used to separate the central part of the atomic gas jet and form the atomic beam which is then injected into the sextupole magnets. The skimmer has a cone shape and its central orifice is $6 \mathrm{~mm}$ in diameter. The distance between the dissociator nozzle and the skimmer orifice is equal to $30 \mathrm{~mm}$. The sextupole magnet system consists of an assembly of three permanent sextupoles

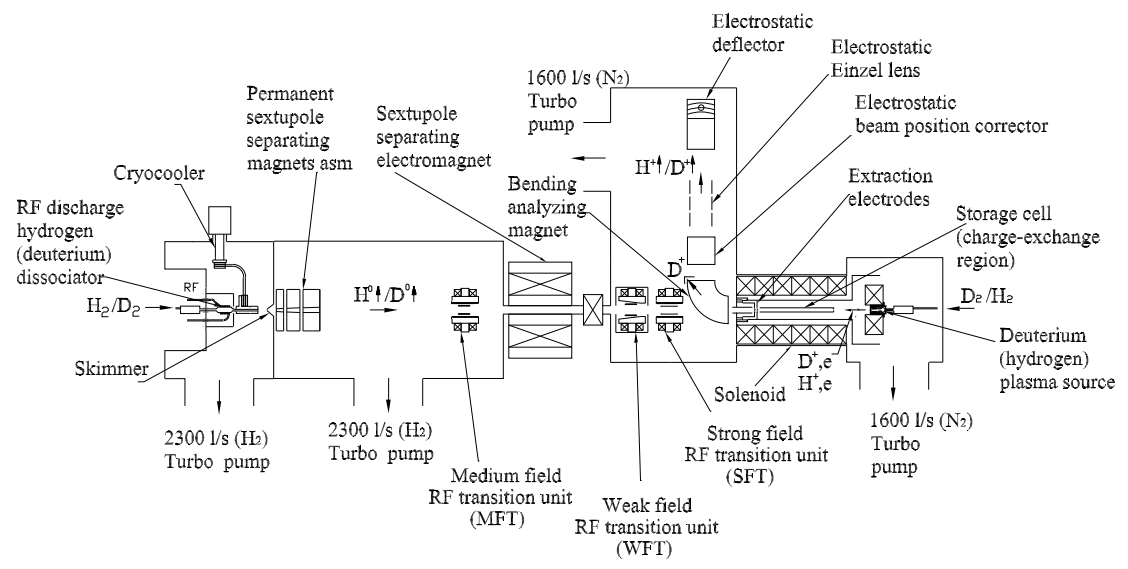

Figure 1: Schematic diagram of the SPI

and one electromagnet sextupole. The sextupole magnet parameters are presented in [5]. The system of high frequency transition (HFT) units includes a medium field transition unit (MFT), which is installed between the assembly of permanent sextupole magnets and the electromagnet sextupole, weak field (WFT) and strong field (SFT) transition units which are installed downstream the electromagnet sextupole. The hydrogen atoms are polarized in the result of WFT $(1 \rightarrow 3)$ and 
SFT $(2 \rightarrow 4)$ initiated by means of RF method. Thus the proton vector polarization: -1 and +1 is provided by these RF transitions. For polarized deuterium the usage of MFT, WFT and SFT will allow the atomic beam to organize the deuteron vector polarization between +1 and -1 and tensor polarization between +1 and $-2[5,6]$. The polarized atomic beam is injected into the plasma ionizer where polarized ions are produced in the following charge-exchange reaction:

$$
\begin{gathered}
H^{0} \uparrow+D^{+} \rightarrow H^{+} \uparrow+D^{0} \\
D^{0} \uparrow+H^{+} \rightarrow D^{+} \uparrow+H^{0} .
\end{gathered}
$$

Plasma ions will undergo charge-exchange collisions with the polarized atoms and thermal energy polarized ions are produced in the region where the polarized atomic beam overlaps with the plasma jet. Radial confinement of the polarized ions is provided by the magnetic field of the solenoid. Then the polarized ions move slowly under the influence of the weak electric field in plasma to the electrode extraction system where they are accelerated to the energy of up to $25 \mathrm{keV}$ together with unpolarized plasma ions. The extracted $25 \mathrm{keV}$ ion beams are separated from unpolarized ions while passing through the $90^{\circ}$ bending magnet. The unpolarized ion beam current is registered by using the ion beam collector downstream the bending magnet. The polarized beam comes out from the magnet in the vertical direction, passes through the electrostatic Einzel lens and then is deflected by a spherical electrostatic mirror into the horizontal plane. The spin direction of the polarized ion beam remains unchanged. At the source exit the polarized ion beam passes through a spin precessor which rotates the spin in the vertical direction. This allows one to acheave the required vertical spin orientation for polarized protons or deuterons.

\section{Tests of the source}

The atomic beam section (ABS) has been tested at INR RAS [6]. The intensity of the polarized atomic hydrogen beam $50 \mathrm{~cm}$ downstream the sextupole electromagnet was measured with the time-of-flight mass-spectrometer and found to be equal to $1.5 \times 10^{17} \mathrm{~s}^{-1} \mathrm{~cm}^{-2}$. The efficiency of HFT was determined by placing a HFT between the assembly of permanent sextupole magnets and electromagnet sextupole. Efficiencies of WFT and MFT for deuterium were determined from these measurements and turned out to be close to $0.95 \pm 0.03$ [6]. First the plasma ionizer was tested at the JINR test-bench without a storage cell. The efficiency of conversion of polarized atoms into polarized ions was proportional to the plasma density in the charge-exchange region and, hence, to the current of the unpolarized ions extracted from the ionizer. The plasma was produced by an arc-discharge plasma source with a cold cathode 0perating in the pulsed mode. The longitudinal magnetic field of $\sim 500 \mathrm{G}$ in the source was produced by the plasma source coil. Molecular deuterium (hydrogen) is injected into the plasma source by a pulsed electromagnetic gas valve similar to the one used for the dissociator. An ignition voltage pulse with the amplitude of $6 \mathrm{kV}$ and duration of about $40 \mu \mathrm{s}$ was applied between the cold cathode and the gas valve body to initiate arc-discharge in the source followed by the $150 \mu$ s voltage pulse applied between the cathode and anode of the source which produces arc-discharge with the current of up to $300 \mathrm{~A}$ peak. The deuterium (hydrogen) plasma generated in the source flows out of the source into vacuum through the hole $4 \mathrm{~mm}$ in diameter in the anode and then it is injected into the ionizer solenoid with 
the longitudinal magnetic field of $\sim 1 \mathrm{kG}$ formed by the ionizer solenoid. The ionizer solenoid coils are at the ground potential while the plasma jet inside the solenoid should be at high voltage positive potential relatively the ground to carry out possible acceleration of ions. The cylindrical screen insulated relatively the ground is installed inside the solenoid to ensure the plasma injection into the region which is at high voltage potential. The screen is connected electrically to the anode of the plasma source.

A three electrode extraction system has been installed to test the ionizer without the storage cell. The first electrode and the second one are gridded with a distance between the grids of $4 \mathrm{~mm}$. The extraction electrode aperture is $17 \mathrm{~mm}$. The first electrode is under the plasma source anode potential while the second electrode is under $-1 \mathrm{kV}$ potential (DC) relatively the ground. The third electrode of a cylindrical form has the internal diameter of $20 \mathrm{~mm}$ and is simply grounded. The potential of the plasma source anode varies up to $25 \mathrm{kV}$ to provide extraction of ions from the plasma region. The negative potential of the second electrode produces a trap for secondary electrons which compensate the space charge of the intense unpolarized ion beam.

The $25 \mathrm{kV}$ pulse duration can be varied in the range of 50-500 $\mu$ s and repetition rate up to $1 \mathrm{~Hz}$. The pulse vertex is stabilized with accuracy of $\pm 0.3 \%$ by means of the feed-back system. The power supply source has been designed and built at INR RAS. Figure 2 shows typical oscillograms characterizing the ionizer operation. The polarized proton beam with the peak current of $1.4 \mathrm{~mA}$

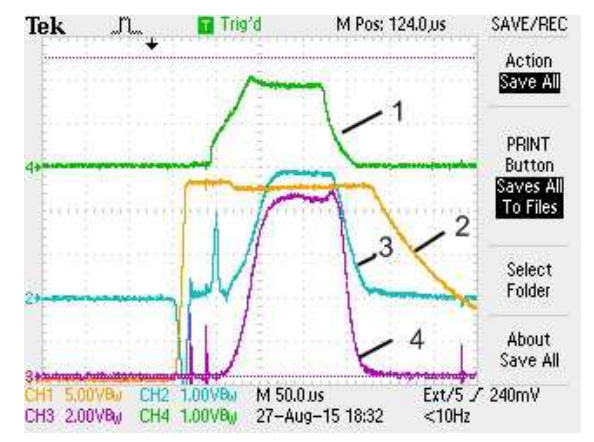

Figure 2: The oscillograms characterizing the ionizer operation: green (1) - the discharge current pulse of the plasma source with the peak current of $190 \mathrm{~A}$, yellow (2) - the beam extraction voltage with the peak value of $23 \mathrm{kV}$, blue (3) - the current of extraction electrodes with the peak value of $560 \mathrm{~mA}$, purple (4) - the unpolarized deuterium ion current recorded downstream the analyzing magnet (the peak value is $170 \mathrm{~mA}$ )

has been recorded downstream the bending magnet. It was registered as the result of the difference in the ion beam peak current with the atomic beam "on" and "off".

\section{Plans}

A storage cell is planned to be used in the charge-exchange region to increase the density of the polarized atom beam. This method has been tested at INR RAS [3] and the polarized proton beam with peak current of $11 \mathrm{~mA}$ and polarization of $80 \%$ has been obtained by means of this method [4].

The HFTs determine nuclear polarization of ions but their efficiency can be influenced by the external magnetic field. For this reason it is important to control the efficiency of HFTs directly in 
their operating mode. To carry out this control we plan to use a Brei-Rabi polarimeter consisting of two multipole magnets and a TOF mass-spectrometer. A schematic diagram of the ABS components and of the above polarimeter is shown in Figure 3. Two permanent multipole magnets will

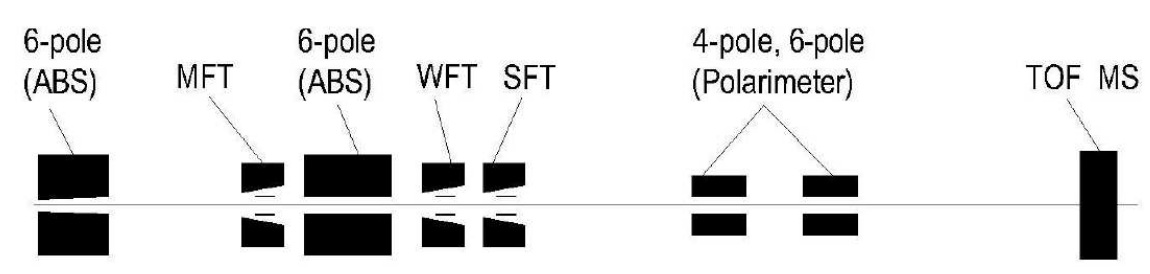

Figure 3: Schematic diagram of the ABS with a Breit-Rabi polarimeter

be used in the Breit-Rabi polarimeter. Their parameters were determined by means of race-tracing calculations. The first magnet is a quadrupole with the aperture of $24 \mathrm{~mm}$ and length of $130 \mathrm{~mm}$. The second magnet is a sextupole with the aperture of $24 \mathrm{~mm}$ and length of $150 \mathrm{~mm}$. The maximum magnetic field in the operation area of first magnet is expected to be $1.68 \mathrm{~T}$. In the working area of second magnet, maximum field is $1.61 \mathrm{~T}$. Now the magnets are under construction.

An example of the calculations of atom trajectories in the source made with the Breit-Rabi polarimeter is shown in Figure 4, 5. It is seen from the results of the calculations that if no HFTs are "on" in the ABS (Figure 4) then the atoms focused by the ABS are refocused by the multipole magnets of the Breit-Rabi polarimeter. If one of the HFTs is "on" then the corresponding part of the atoms undergoing the transition between the spin states (with change of their electron spin state in the strong magnetic field from $m_{j}=-1 / 2$ to $m_{j}=+1 / 2$ ) will be defocused by the multipole magnets of the Breit-Rabi polarimeter (Figure 5). Measurements of the relative change of the polarized atomic beam density by the TOF mass-spectrometer with HFTs "off" and "on", will allow one to determine the efficiency of HFT of the ABS.

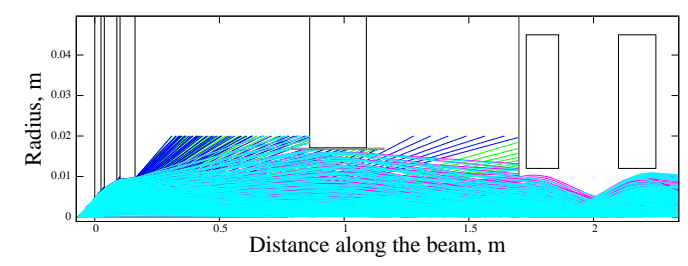

Figure 4: Trajectories of atoms in the ABS and the Breit-Rabi polarimeter. HFTs are "off"

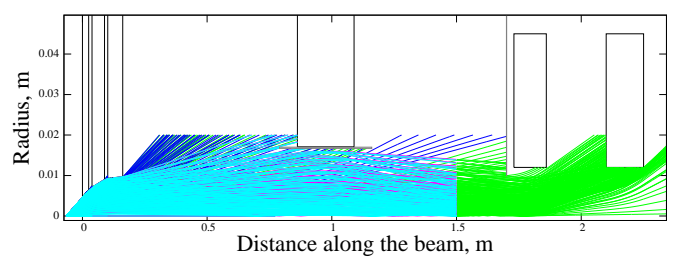

Figure 5: Trajectories of atoms in the ABS and the Breit-Rabi polarimeter. HFTs are "on"

\section{Conclusions}

The polarized ion source for the JINR accelerator complex is now under testing at the JINR test bench. The ABS part and the plasma ionizer operation have been tested and found to be satisfactory. The polarized proton beam of $1.4 \mathrm{~mA}$ peak has been produced with the free atomic beam in the charge-exchange region of the source. It is planned to continue tests with a storage cell installed into the ionizer. The HFT units will be retuned in their operating mode by means of the Breit-Rabi polarimeter. 


\section{References}

[1] Trubnikov G., Agapov N., Brovko O. et al, 2014 Proc. of IPAC2013(Shanghai, China) ISBN 978-3-95450-122-9 p 1343.

[2] Belov A. S., Esin S. K., Kubalov S. A., Kuzik V. E., Stepanov A. A. and Yakushev V. P., 1987 Nucl. Instrum. and Meth. In Phys. Res. A255 442.

[3] Belov A. S., Esin S. K., Netchaeva L., P., Klenov V. S., Turbabin A. V. and Vasil'ev G. A., 1998 Proc. Int. Workshop on Polarized Gas Targets and Polarized Beams (Urbana IL, USA, 1997) eds. Ro J. Holt and M. A. Miller, AIP Conf. Proc., 421, p 362.

[4] Belov A. S., Esin S. K., Netchaeva L. P., Turbabin A. V. and Vasil'ev G. A., 1999 Proc. Int. Symp. on High Energy Spin Physics (Protvino, Russia, 1998) ed. N. E. Tyurin e al, (Singapore: World Scientific) p 22.

[5] Derenchuk V. P. and Belov A. S., Proceedings of the 2001 PAC, Chicago, USA, p 2093.

[6] Fimushkin V. V., Kovalenko A. D., Kutuzova L. V., Prokofichev Yu. V., Belov A. S., Turbabin A. V. and Zubets V. N., 2014 Proc. 20th Int. Symp. on High Energy Spin Physics (Dubna, Russia, 2012) PEPAN 45 part 1, p 297. 